Check for updates

Cite this: RSC Adv., 2017, 7, 21050

Received 6th January 2017

Accepted 3rd April 2017

DOI: $10.1039 / \mathrm{c} 7 \mathrm{ra00212b}$

rsc.li/rsc-advances

\section{A dansyl-based fluorescent probe for the highly selective detection of cysteine based on a d-PeT switching mechanism $\dagger$}

\author{
Yudong Xiao, ${ }^{\text {b } Y u j i n ~ G u o, ~}{ }^{a}$ Ruili Dang, ${ }^{a}$ Xin Yan, ${ }^{c}$ Pengfei Xu (D) *a and Pei Jiang ${ }^{\star a}$ \\ A novel fluorescent probe, DN-C, for detection of cysteine (Cys) based on a d-PeT switching mechanism is \\ reported. In the presence of Cys, the probe exhibits a turn-on fluorescence signal and nearly 28-fold \\ fluorescence intensity enhancement. The cellular imaging experiment indicated the DN-C possess \\ desirable cell permeability for biological applications.
}

As an important amino acid, cysteine (Cys) plays a crucial role in protein synthesis, detoxification, and metabolism., ${ }^{\mathbf{1} 2}$ Cys is a precursor amino acid of glutathione (GSH) and both are taken in by the human body through food or are formed as a metabolic product of homocysteine (Hcy). Deficiency of Cys is associated with many syndromes, including slowed growth, hair depigmentation, lethargy, liver damage, muscle and fat loss, skin lesions, and weakness. ${ }^{3}$ Its significant biological role explains the considerable contemporary effort devoted to the development of an efficient method for the detection and quantification of Cys under physiological conditions.

A number of analytical methods for the detection of Cys have been developed using high-performance liquid chromatography (HPLC), capillary electrophoresis, electrochemical assay, UV/Vis, FTIR, mass, and fluorescence spectroscopy. ${ }^{\mathbf{4 1 0}}$ Among these methods, fluorescence probes are more desirable due to its high selectivity, low detection limit, fast response and great potential for bioimaging. ${ }^{11}$ Probes rely on fluorescence quenching suffer from inherent drawbacks including low signal-to-noise ratio and nonspecific quenching, so that "turnon" type fluorescence probes are preferred. ${ }^{12,13}$ To date, various types of organic reactions, such as cyclization reaction and Michael addition, have been employed to design fluorescencebased probes. Nevertheless, only a few probes are able to discriminate between Cys, Hcy, and GSH. ${ }^{\mathbf{1 4 - 1 6}}$ Thus, further development of synthetically simple and practical probes for selective detection of Cys is still highly required because of the

aInstitute of Clinical Pharmacy \& Pharmacology, Jining First People's Hospital, Jining Medical University, Jining 272000, China. E-mail: jiangpeicsu@sina.com; pengfeixucsu@outlook.com

${ }^{b}$ Department of Radiology, The Second Xiangya Hospital of Central South University, Changsha 410010, China

'Research Center for Drug Discovery, School of Pharmaceutical Sciences, Sun Yat-sen University, 132 East Circle at University City, Guangzhou, 510006, China

$\dagger$ Electronic supplementary information (ESI) available. See DOI: $10.1039 / \mathrm{c} 7 \mathrm{ra00212b}$ broad functional properties of Cys within complex biological systems.

Dansyl fluorophore is widely used in amino acids modification, protein sequencing and amino analysis, because of its high fluorescence quantum yields and large Stokes shift. To date, a number of dansyl based probes for the detection of metal ion have been reported, mainly based on the electron transfer effect (ET) between metal ion and the excited dansyl fluorophore in the coordination state. ${ }^{17-21}$ Obviously, this strategy isn't suit for the design of probes for biological molecules such as Cys. To the best of our knowledge, dansyl based fluorescent probes for Cys are rare. In the fact, there is only one reported dansyl based fluorescent probe for Cys by Wang group. ${ }^{22}$ In this work, we introduced a new strategy to design dansyl based probes and realized the detection of Cys in vivo.

Our strategy to design a dansyl based fluorescence probe for cellular Cys relies on modulating donor-excited photoinduced electron transfer (d-PeT) process from the excited fluorophore to a strong electron-withdrawing group, which can switch the emission signal. ${ }^{2324}$ Thus, the core of the strategy is depending on the carefully choosing a Cys responding group, which can simultaneously the PET acceptor. On other hand, it has been known for several years that the conjugate addition of Cys to acrylates will generate the corresponding thioether, which can further undergo an intramolecular cyclization to yield 3carboxy-5-oxoperhydro-1,4-thiazepine. Meanwhile, acryloyl group has low LUMO energy level and can serve as the PET acceptor. Therefore, $\alpha, \beta$-unsaturated carbonyl moiety was selected as the responding group and signal modulator for probe DN-C.

The synthetic route for DN-C was shown in Scheme 1. DN-C was readily prepared in two convenient steps under facile conditions with high yield. The DN was prepared starting from dansyl chloride and 3-aminophenol in acetonitrile at room temperature. The reaction of $\mathbf{D N}$ and acryloyl chloride in anhydrous acetonitrile afforded DN-C with a high yield of $87 \%$. 


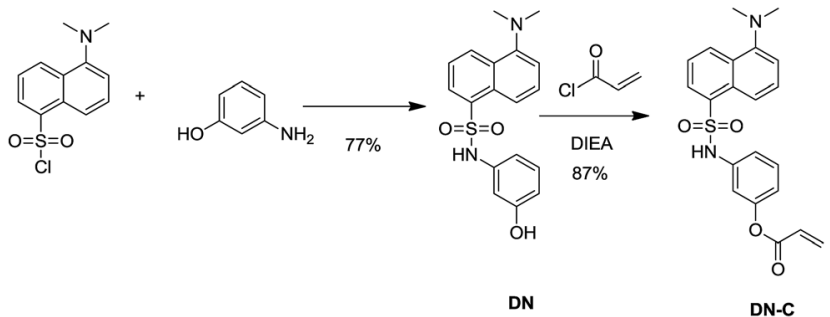

Scheme 1 The synthetic route of DN-C.

The structure of DN-C was fully characterized by ${ }^{1} \mathrm{H}$ NMR, ${ }^{13} \mathrm{C}$ NMR and MS.

Spectral properties of DN and DN-C were first examined in a mixed solution of $\mathrm{CH}_{3} \mathrm{CN}: \mathrm{PBS}(50: 50, \mathrm{v} / \mathrm{v}, \mathrm{pH}=7.4,10 \mathrm{mM})$. As shown in Fig. 1, DN exhibits a robust emission peak with maximum at $545 \mathrm{~nm}$. However, the emission intensity of DN-C decline markedly. The suppression of fluorescence emission may attribute to the occurrence of donor-excited photoinduced electron transfer (d-PeT) process. The process of d-PeT involves transfer of one electron from the excited donor unit to the lowest unoccupied molecular orbital (LUMO) of the electrondeficient unit. To verify this hypothesis, the HOMO and LUMO energy levels of dansyl group and acryloyl group were calculated by suite of Gaussian 09 programs [density functional theory (DFT) time-dependent density function theory (TD-DFT)] at B3LYP/6-31G basis sets. The calculation result indicated that the LUMO energy level of acryloyl group is in the range of between the HOMO and LUMO energy levels of dansyl group, which is appreciate for d-PeT process (Fig. 2). The electron transfer from the dansyl framework (PET donor) to the acryloyl group (PET acceptor) weakened the fluorescence of the primordial fluorophore. Thus, the dansyl fluorophore will be in the off state. Once removal of the d acryloyl group, like the structure of DN, the d-PeT progress will disappear and the fluorescence will restore.

Subsequently, we performed the fluorescence titration studies of DN-C towards Cys in a mixed solution of $\mathrm{CH}_{3} \mathrm{CN}$ : PBS $(50: 50, \mathrm{v} / \mathrm{v}, \mathrm{pH}=7.4,10 \mathrm{mM})$. The absorption and fluorescence spectra of the solution of DN-C treated with a series of Cys (0 to

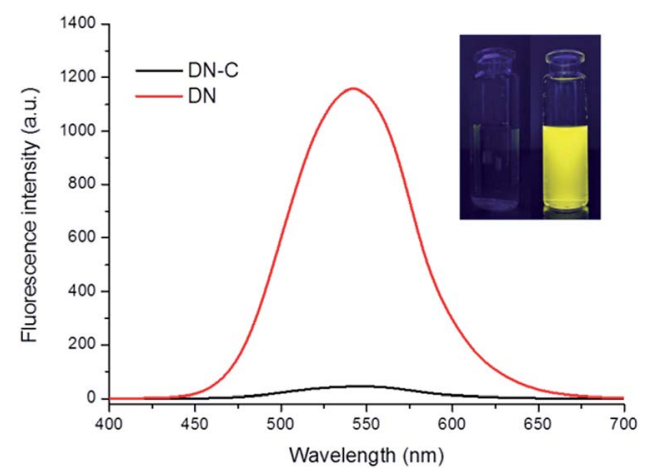

Fig. 1 Fluorescence spectra of DN and DN-C (10 $\mu \mathrm{M})$. Inset: fluorescence changes of probe DN-C upon the addition of Cys $(100 \mu \mathrm{M})$ under excitation with UV light (365 nm).

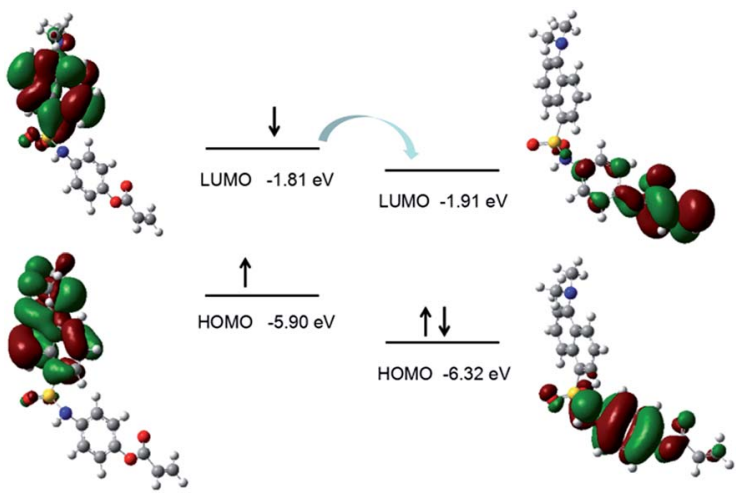

Fig. 2 Frontier molecular orbitals (MOs) of dansyl group and acrylate group. In the ball-and-stick model, carbon, oxygen, sulfur and nitrogen atoms are colored in gray, red, yellow and blue, respectively.

$100 \mu \mathrm{M})$ were recorded. As shown in Fig. 3, the free DN-C displayed quite weak fluorescence. With the addition of Cys, the fluorescence intensity of DN-C increased significantly at $545 \mathrm{~nm}$ due to specifically trigger the cleavage of acryloyl group by Cys. As shown in Fig. 4, the emission intensities of $545 \mathrm{~nm}$ increased by nearly 28 -fold. In addition, the increase in fluorescence intensity is in a concentration dependent manner and exhibits good linear correlation with the amount of Cys $\left(0-30 \mu \mathrm{M}, R^{2}=\right.$ 0.98412). A maximal fluorescent signal is achieved in the presence of only 5 equiv. of Cys and the detection limit of DN-C for Cys was determined as $13 \mathrm{nM}$, indicating that the probe is sensitive enough to Cys and might be suitable for detecting endogenous Cys in biological samples.

To evaluate the selectivity of DN-C for Cys, we measured the fluorescence intensity changes for various analytes upon addition of excess guests (Fig. 5). The fluorescence intensity of DN-C was highly enhanced only by the addition of Cys. Other amino acids, such as Hcy, GSH, Gly, Phe, Ser, Glu, Lys, Arg, His, Ala, Gln, Met, and Tyr, did not cause any significant changes in the fluorescence emission intensity of $545 \mathrm{~nm}$. This result indicated that probe DN-C exhibited remarkably higher selectivity for Cys over Hcy, GSH and other amino acids at neutral conditions.

Furthermore, the practical utilities of DN-C was investigated for fluorescent imaging of intracellular Cys in living T-47D

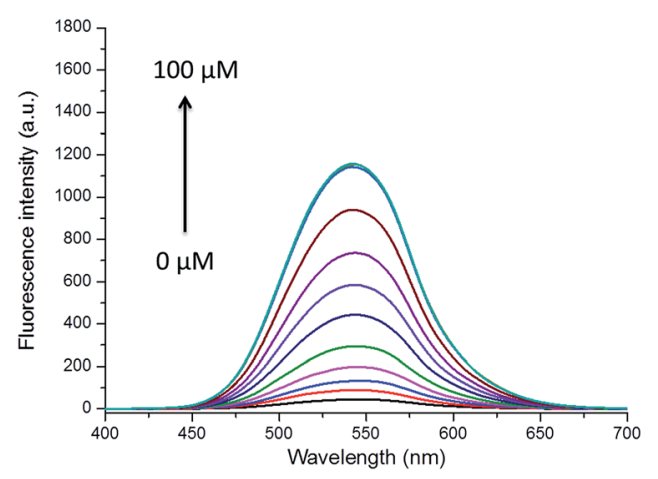

Fig. 3 Emission spectra of DN-C $(10 \mu \mathrm{M})$ in a mixed solution of $\mathrm{CH}_{3} \mathrm{CN}$ : PBS (50:50, v/v, $\left.\mathrm{pH}=7.4,10 \mathrm{mM}\right)$ upon addition of Cys (0$100 \mu \mathrm{M})$ with $\lambda_{\mathrm{ex}}=380 \mathrm{~nm}$. 

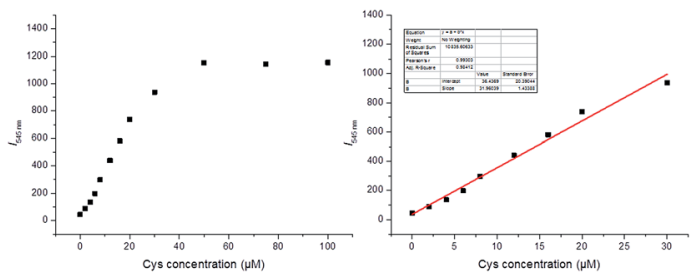

Fig. 4 Plot of emission intensity at $545 \mathrm{~nm}$ as a function of Cys concentration when using DN-C $(10 \mu \mathrm{M})$ in a mixed solution of $\mathrm{CH}_{3}$ $\mathrm{CN}:$ PBS $(50: 50, \mathrm{v} / \mathrm{v}, \mathrm{pH}=7.4,10 \mathrm{mM})$ with $\lambda_{\mathrm{ex}}=380 \mathrm{~nm}$.

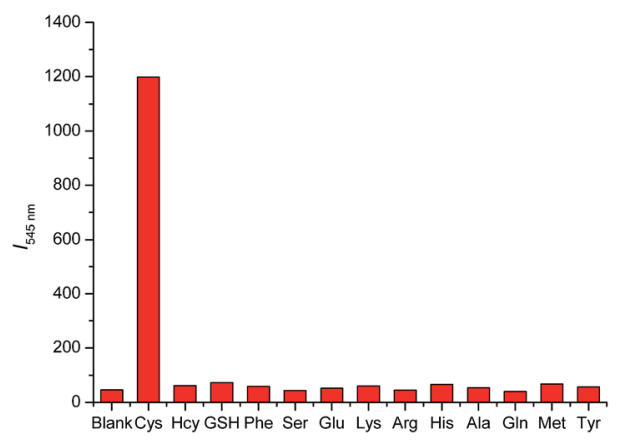

Fig. 5 Fluorescence responses of $\mathrm{DN}-\mathrm{C}(10 \mu \mathrm{M})$ to various analytes in a mixed solution of $\mathrm{CH}_{3} \mathrm{CN}$ : PBS $(50: 50, \mathrm{v} / \mathrm{v}, \mathrm{pH}=7.4,10 \mathrm{mM})$ with $\lambda_{\mathrm{ex}}=380 \mathrm{~nm}$.

human breast duct carcinoma cells (T-47D human breast duct carcinoma cells were purchased from Abcam). As shown in Fig. 6, in the control experiment, when $N$-ethylmaleimide (NEM), a well know thiol trapping reagent, was added to the cell culture prior to the addition DN-C $(10 \mu \mathrm{M})$, faint fluorescence was observed in the green channel (b). However, when T-47D cells were only incubated with DN-C $(10 \mu \mathrm{M})$ for $30 \mathrm{~min}$, bright green fluorescence could be observed (d). These results
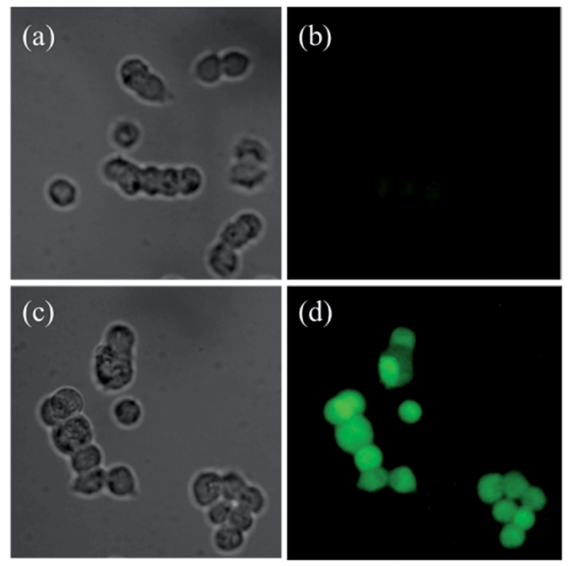

Fig. 6 Top: T-47D human breast duct carcinoma cells were preincubated with $500 \mathrm{mM}$ NEM for $50 \mathrm{~min}$ and then treated with $10 \mu \mathrm{M}$ of DN-C for 30 min; bottom: T-47D human breast duct carcinoma cells were treated with $10 \mu \mathrm{M}$ of DN-C for $30 \mathrm{~min}$ ((a and c), bright-field image; ( $b$ and $d)$, fluorescence images. The excitation wavelength is 330-400 nm and the emission was collected in green channel). demonstrated that DN-C possesses good membrane permeability and is able to detect intracellular Cys in living cells.

\section{Conclusions}

In conclusion, we have designed and synthesized selective and sensitive fluorescent probe DN-C for Cys based on dansyl chromophore. The probe exhibits a turn-on fluorescence signal for responding Cys via d-PeT switching mechanism. It has a selectivity and sensitivity for in vitro Cys over other various biologically relevant species, and could detect Cys in living cells. Therefore, we anticipate that the superior properties of DN-C will make it of potential use in real-time Cys monitoring, and as an efficient research tool for the study on Cys related molecular events in biological systems.

\section{Acknowledgements}

The study was supported by the National Natural Science Foundation of China (No. 81602846) and Natural Science Foundation of Shandong Province (No. ZR2016HQ21).

\section{References}

1 K. G. Reddie and K. S. Carroll, Curr. Opin. Chem. Biol., 2008, 12, 746-754.

2 C. E. Paulsen and K. S. Carroll, Chem. Rev., 2013, 113, 46334679.

3 S. Shahrokhian, Anal. Chem., 2001, 73, 5972-5978.

4 N. Ercal, P. Yang and N. Aykin, J. Chromatogr. B: Biomed. Sci. Appl., 2001, 753, 287-292.

5 P. Ryant, E. Dolezelova, I. Fabrik, J. Baloun, V. Adam, P. Babula and R. Kizek, Sensors, 2008, 8, 3165-3182.

6 O. Rusin, N. N. St Luce, R. A. Agbaria, J. O. Escobedo, S. Jiang, I. M. Warner, F. B. Dawan, K. Lian and R. M. Strongin, J. Am. Chem. Soc., 2004, 126, 438-439.

7 X. Guan, B. Hoffman, C. Dwivedi and D. P. Matthees, $J$. Pharm. Biomed. Anal., 2003, 31, 251-261.

8 D. Potesil, J. Petrlova, V. Adam, J. Vacek, B. Klejdus, J. Zehnalek, L. Trnkova, L. Havel and R. Kizek, J. Chromatogr. A, 2005, 1084, 134-144.

9 Y. Sato, T. Iwata, S. Tokutomi and H. Kandori, J. Am. Chem. Soc., 2005, 127, 1088-1089.

10 Y. Zhou and J. Yoon, Chem. Soc. Rev., 2012, 41, 52-67.

11 X. Chen, Y. Zhou, X. Peng and J. Yoon, Chem. Soc. Rev., 2010, 39, 2120-2135.

12 H. S. Jung, K. C. Ko, G. H. Kim, A. R. Lee, Y. C. Na, C. Kang, J. Y. Lee and J. S. Kim, Org. Lett., 2011, 13, 1498-1501.

13 X. Zhang, X. Ren, Q. H. Xu, K. P. Loh and Z. K. Chen, Org. Lett., 2009, 11, 1257-1260.

$14 \mathrm{H}$. Wang, G. Zhou, H. Gai and X. Chen, Chem. Commun., 2012, 48, 8341-8343.

15 X. Yang, Y. Guo and R. M. Strongin, Org. Biomol. Chem., 2012, 10, 2739-2741.

16 Z. Guo, S. W. Nam, S. Park and J. Yoon, Chem. Sci., 2012, 3, 2760-2765. 
17 K. H. Jung, E. T. Oh, H. J. Park and K. H. Lee, Biosens. Bioelectron., 2016, 85, 437-444.

18 P. Xie, F. Guo, L. Wang, S. Yang, D. Yao and G. Yang, J. Fluoresc., 2015, 25, 319-325.

19 C. Rivas, G. J. Stasiuk, M. Sae-Heng and N. J. Long, Dalton Trans., 2015, 44, 4976-4985.

20 L. J. Ma, J. Liu, L. Deng, M. Zhao, Z. Deng, X. Li, J. Tang and L. Yang, Official Journal of the European Photochemistry Association and the European Society for Photobiology, Photochem. Photobiol. Sci., 2014, 13, 1521-1528.
21 J. Piao, J. Lv, X. Zhou, T. Zhao and X. Wu, Spectrochim. Acta, Part A, 2014, 128, 475-480.

22 H. Zhang, P. Wang, Y. Yang and H. Sun, Chem. Commun., 2012, 48, 10672-10674.

23 X. Zhang, L. Chi, S. Ji, Y. Wu, P. Song, K. Han, H. Guo, T. D. James and J. Zhao, J. Am. Chem. Soc., 2009, 131, 17452-17463.

24 S. Ji, J. Yang, Q. Yang, S. Liu, M. Chen and J. Zhao, J. Org. Chem., 2009, 74, 4855-4865. 